\title{
Asymmetries arising from the space-filling nature of vascular networks
}

\author{
David Hunt ${ }^{1}$ and Van M. Savage ${ }^{1,2,3}$ \\ ${ }^{1}$ Department of Biomathematics, University of California at Los Angeles, Los Angeles, California 90095, USA \\ ${ }^{2}$ Santa Fe Institute, Santa Fe, New Mexico 87501, USA \\ ${ }^{3}$ Department of Ecology and Evolutionary Biology, University of California at Los Angeles, Los Angeles, California 90095, USA
}

(Received 18 August 2015; revised manuscript received 1 March 2016; published 9 June 2016)

\begin{abstract}
Cardiovascular networks span the body by branching across many generations of vessels. The resulting structure delivers blood over long distances to supply all cells with oxygen via the relatively short-range process of diffusion at the capillary level. The structural features of the network that accomplish this density and ubiquity of capillaries are often called space-filling. There are multiple strategies to fill a space, but some strategies do not lead to biologically adaptive structures by requiring too much construction material or space, delivering resources too slowly, or using too much power to move blood through the system. We empirically measure the structure of real networks (18 humans and 1 mouse) and compare these observations with predictions of model networks that are space-filling and constrained by a few guiding biological principles. We devise a numerical method that enables the investigation of space-filling strategies and determination of which biological principles influence network structure. Optimization for only a single principle creates unrealistic networks that represent an extreme limit of the possible structures that could be observed in nature. We first study these extreme limits for two competing principles, minimal total material and minimal path lengths. We combine these two principles and enforce various thresholds for balance in the network hierarchy, which provides a novel approach that highlights the tradeoffs faced by biological networks and yields predictions that better match our empirical data.
\end{abstract}

DOI: 10.1103/PhysRevE.93.062305

\section{INTRODUCTION}

The vital functions of the cardiovascular system are the distribution of oxygen and nutrient resources throughout the body, as well as the collection and filtration of waste by circulating blood. Transfer of resources and waste occurs primarily at the capillary level via diffusion through nearby tissue. Consequently, this smallest level of the network must reach all living cells in order to maintain them, filling the entire space of the body. In models developed by Krogh for effective diffusion of oxygen [1], cells cannot survive beyond a maximum distance from a capillary. This defines a service volume of cells that is associated with each capillary, which has a typical size that has been observed to vary across species based on cellular metabolic rate $[2,3]$. The constraint on maximum distance from capillaries necessitates that the final levels of the cardiovascular network are also space-filling throughout the body. In this paper we investigate the relation between this space-filling property and basic optimization principles such as the minimization of costs from construction material and pumping power. Specifically, we highlight how this relation influences the asymmetries in sizes and flow rates of sibling segments as measured in empirical data.

A central focus of our investigation of cardiovascular systems is the space-filling properties of networks, but these properties are also of great interest in many other contexts. General space-covering hexagonal patterns appear in nature in the cell structure of beehives as well as in economic theories for market areas [4]. Trees (the woody, perennial plants) have been studied for both how forests fill an area [5], as well as how the vascular

Published by the American Physical Society under the terms of the Creative Commons Attribution 3.0 License. Further distribution of this work must maintain attribution to the author(s) and the published article's title, journal citation, and DOI. structure within an individual plant determines the hydraulics of resource delivery [6,7]. Apollonian networks [8] describe the space-filling packing of spheres of various sizes, which are similar in the cardiovascular system to considering the volumes of different subtrees of the network. Efficiently filling space in two dimensions is important for information visualization [9]. In addition to these applications, Kuffner and LaValle study space-filling tree networks (i.e., networks that branch and have no closed loops) to determine a route from one location to another [10], but without the biological constraints that we impose here. For cardiovascular networks, this motion planning is analogous to how the vascular structure routes blood. Efficient routing of blood through a hierarchy is central to models that investigate allometric scaling of metabolic rate with body mass [2,3,11-14], which build on metabolic scaling by Kleiber [15]. Determining specific space-filling strategies will inform these models to better describe the cardiovascular system.

Developmental processes (i.e., growth) as well as evolutionary pressures, such as efficiency in material and energy use, shape the structure of cardiovascular networks. For instance, the system must be robust to developmental processes and damage that cause changes in vessel lengths and the number of hierarchical levels [16,17]. We investigate optimal space-filling networks without invoking an explicit growth or developmental process, as do most models of vascular networks. Nevertheless, our choices are consistent with the present knowledge of vascular development. A key feature of vascular development is that the growth of individual segments and branching are triggered by signaling (e.g., by VEGF [18]), effectively guiding angiogenesis and the eventual location of capillaries. Remarkably, such guidance leads to an efficient, global, space-filling network built from local chemical concentrations. This is all the more remarkable because general strategies for efficiently filling a volume or surface are nontrivial, especially when the distribution system must reliably deliver blood at each stage of development. Because the vasculature must be efficient throughout all 
stages of growth, we focus on the static properties of optimal space-filling networks.

In this paper, we explain how the challenges of constructing an efficient space-filling network can lead to the asymmetric structures in both the local relations of segments, as well as in the paths from the heart to each service volume that we observe in empirical data. At the local level, the ratio of lengths between parent and child segments may vary across the network, deviating from strict self-similarity. Additionally, sibling segments may vary in length, which we call asymmetric branching. Within our numerical method, these features arise as a result of optimizing branch-point positions relative to adjacent branch points across the network. Variation in the length and number of levels in paths means that the network is also not symmetric or balanced in these global properties. By allowing these asymmetries and explicitly ensuring space-filling structure, we expand other models that are strictly balanced in network hierarchy and perfectly symmetrical in local quantities.

Asymmetries observed in real systems motivate our investigation of the space-filling properties and asymmetries in cardiovascular networks. These observations show that such networks have a tendency for the lengths of sibling segments to be distributed less symmetrically than is the case for radii. The empirical data in Sec. V come from two types of sources. Images collected through microtomography of mouse lung comprise one data set. The mice were part of a study with different manipulations of matrix GLA protein (which causes the vasculature to be under- or over-branched [19]), but we focus on the wild-type specimen for our analysis. The other data set, collected through MRI, excludes the lungs and instead focuses on the central vasculature in the human head and torso [20]. We utilize the custom software Angicart [20] to analyze these two distinct vascular data sets. Because of the noninvasive nature of these data acquisitions and analysis techniques, future studies have the opportunity to track the development of cardiovascular systems as individual organisms grow and age, including repair after the system incurs damage (i.e., wound healing). Such studies can elucidate the effects that patterns of growth and changes from damage have on the final, mature state of the network.

In this paper, we study the optimization principles that correspond to evolutionary pressures for efficiency in material cost and power loss. Our focus is the influence of space-filling patterns on length asymmetry distributions without the explicit inclusion of radius information. The list of candidate networks includes all possible hierarchical (topological) connections between the heart and all capillaries. For each hierarchy and unique permutation of pairings between terminal vessels and service volumes [see Fig. 1(d)], we must determine the positions of the branch points. The combination of the hierarchy, service-volume pairings, and branch-point positions defines the configuration of a candidate network. For these reasons, we must search through many candidate configurations to determine the most efficient structure. We quantify the fitness

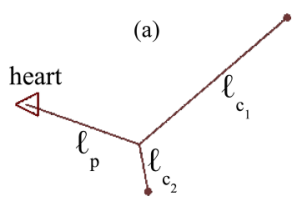

(d)
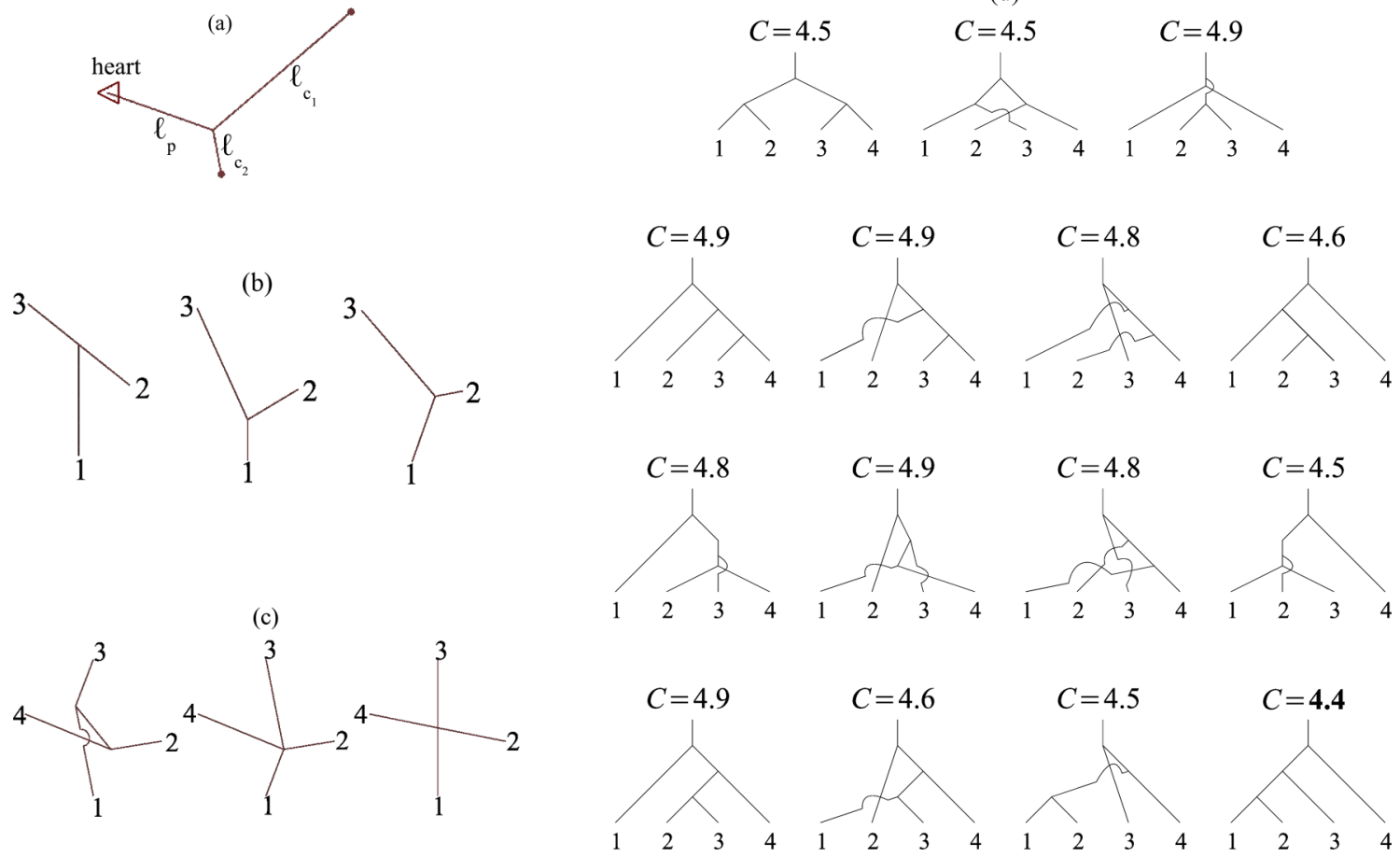

FIG. 1. (a) Schematic of the simplest bifurcating tree network, showing the heart (hollow red triangle) and two service volumes (filled brown circles) with labels for the lengths of each associated segment. (b) Local optimization of branch point position. Three possible locations for a bifurcation junction. The rightmost configuration shows the Fermat point of $\triangle 123$ that minimizes the sum of segment lengths. From left to right, the sum of lengths are 2.38, 2.19, and 2.08. (c) The two distinct bifurcations (left) collapse to a single trifurcation (center) and set to the geometric median of the four endpoints (right). From left to right, the sum of lengths are 2.50, 2.09, and 2.02. (d) Global optimization via search through the space of hierarchies. Comparing $C$ (a measure of some length property of the network) for each of the 15 configurations for four service volumes shows that the bottom right configuration is optimal with respect to $C$. The relative order and position of both the tips and branch points in (d) do not correspond to relative positions in space. 
of each candidate network using individual segment lengths between branch points as well as full path lengths between each capillary and the heart.

To perform a reliable comparison between candidate configurations, it is crucial to determine branch-point positions in a consistent way. We determine these positions iteratively for the entire network in order to identify the global optimum. While the local process of choosing branch points that minimize total vessel lengths (or similar features) is relatively straightforward to iterate over the network, any single branch point and its relation to its neighbors relies indirectly on updates that are applied elsewhere. This dependence emerges from the fact that each end of a vessel is connected to a branch point, which upon repositioning affects the lengths of all vessels that it joins. The uniqueness of the Fermat point - the branch point position that minimizes the sum of the lengths of vessels at a single junction-is already well established (for example, see Ref. [21]). This allows us to carefully construct an algorithm (described in Sec. II C) that reliably relaxes all branch-point positions into the global optimum.

After determining the positions of branch points for a given hierarchy, we compare distinct configurations to find the optimum network. The search through configurations is also a central problem in phylogenetics, where the goal is to construct phylogenies to identify similar groups of species and trace the development of genes through speciation. Even in the case of genes that control biological traits, a loosely analogous space-filling phenomenon emerges in the form of species filling the niches in the environment. With our specific goal of complete spatial covering of network tips, we develop strategies in Sec. IV for exploring the space of hierarchies that are similar to those used on phylogenetic trees.

The organization of the subsequent sections is as follows. In Sec. II, we describe the basic assumptions for our space-filling network model, including the details of the local optimization of branch-point locations and the global paths through the network. In Sec. III, we introduce the specific quantitative network properties that we use to compare the fitness of candidate networks. We introduce the properties of the space of tree hierarchies and our implemented exploration strategies in Sec. IV. In Sec. V, we detail the results from the several layers of optimization that we implement, and we discuss the insights that they offer in Sec. VI.

\section{CONSTRUCTION OF ARTIFICIAL VASCULAR NETWORKS}

To better understand the connection between the local asymmetries of individual vessel lengths and the global constraint on space-filling capillaries, we optimize candidate networks that are embedded in two spatial dimensions (2D) in silico with respect to specific optimization principles. Corroborated by simulations, constructing networks in three spatial dimensions (3D) gives qualitatively similar results but requires much larger numbers of tips that necessitate a greater computational investment. The small quantitative differences between $2 \mathrm{D}$ and 3D are likely explainable because of the extra space (i.e., an extra dimension) to arrange vessels, allowing for an increased number of possible arrangements of neighboring vessels at all levels. Consequently, we focus on 2D networks to achieve configurations with larger numbers of branching levels, ease computation, and obtain the same general results and conclusions. We explore these optimized artificial 2D networks and quantify their branching length asymmetries to compare with our empirical data. Our model's simplification of the cardiovascular network focuses on the lengths of segments as defined by the straight line between adjacent branch points. Reticulated structures occur within leaves to mitigate damage $[16,17]$ and within animals as anastomoses (or pathologically as fistulas). However, we focus on the vast majority of the cardiovascular system that distributes resources through a hierarchical, treelike structure, in which no segments or subtrees rejoin downstream to form closed loops before reaching the capillaries. This is sufficient for the focus of our investigation of the asymmetric, space-filling structure that distributes the resource-rich blood from the heart throughout the body.

The space-filling property of the cardiovascular network constrains the hierarchical structure of the network and the positions of branch points. Here we describe our process for the construction of individual networks and the space of possible networks through the following steps: defining a distribution of space-filling service volumes in the space of the body, identifying all unique hierarchies and pairings between tips of hierarchies and distinct service volumes, and determining the positions of branch points for each hierarchy and pairing.

\section{A. Space-filling service volumes}

Because real systems do not organize or grow on a regular (symmetric, isotropic) grid, we position service volumes randomly within the space they fill. Although the exact location of tips in the adult organism or at intermediate stages of growth is not known during earlier stages of development, the sources of the largest concentrations of VEGF and other signals guide vascular growth and thus effectively define the service volumes before the physical vessels are even present. Construction of service volumes begins by choosing a random point within the body volume that represents the location of a capillary. We then randomly choose other points (capillary locations) so that none lie within a predefined constant distance from another capillary location. After determining a set of capillary locations that span the 2D area, the entire body is partitioned into Voronoi cells fed by the closest capillary. In this way, each capillary becomes associated with a specific service volume, and the sum of the service volumes fills the entire space (see Fig. 3 or 5).

\section{B. Space of hierarchically distinct trees and pairings with service volumes}

Because multiple branching levels connect the service volumes to the heart, there are many possible hierarchical orderings of branching junctions across these different levels. For example, there are two unique hierarchies when there are four service volumes: the top three configurations in Fig. 1(d) are the same perfectly balanced hierarchy, while the remaining trees have the same unbalanced hierarchy. The distinguishing feature is the pairings of tips in the hierarchy to specific service volumes (1-4). 
There are many distinct pairings of terminal tips with the set of fixed service volumes for each bifurcating tree. For four service volumes, there are a manageable 15 unique bifurcating trees [shown in Fig. 1(d)]. Before determining branch-point positions for small networks, we exhaustively search through all possible hierarchies and pairings with service volumes. For large networks, the number of distinct hierarchies and pairings [(2n-3)!! for $n$ distinct service volumes] is prohibitively large, so we sample the space as described in Sec. V B.

We do not disqualify configurations if one vessel path crosses with another (these would likely separate in three dimensions), and there is no exchange of resources or interaction in blood flow at such locations. Crossings are not observed for networks that minimize only total network length without a constraint on hierarchical balance, but they often occur for optimal configurations with a strong constraint on hierarchical balance.

\section{Optimization of branch point positions for a fixed hierarchy and pairing}

We now detail our algorithm for the optimization of the positions of branch points that connect a distribution of service volumes to the heart through a hierarchical network. Within our algorithm, the position of each branch point depends solely on the location of the adjacent branch points in the network. Distant vessels affect each other indirectly, but not through any direct long-range process. Using the limited, local information given by the neighborhood of a branch point, each junction is assigned a uniquely defined position that minimizes the sum of the Euclidean distances to each neighboring junction. This is equivalent to the Fermat point of the triangle formed by the two downstream ends of the child vessels and the one upstream end of the parent vessel [see Fig. 1(b)]. Although it may seem counterintuitive to specify the downstream endpoints before the branching junction is in place, these endpoints correspond to sources of VEGF during growth, allowing us to specify the downstream branch point before the physical vessels or branching are in place. In real systems, the exact branch point location has also been shown to depend on the specific architectural geometry and location of highest shear stress in the parent vessel [22]. This effect might add small corrections to our predictions but is not expected to change the qualitative properties that we observe, especially when averaged over the entire network. The Fermat point of a triangle is a special case of the more general geometric median, the unique point that minimizes the sum of distances to an arbitrary number of other fixed points. We follow the algorithm presented in Ref. [23] to avoid errors in determining the geometric median. Assigning branch-point positions as geometric medians effectively minimizes the construction costs for the local network structure.

We construct our networks from simple bifurcations, but using the Fermat point to assign branch-point positions can lead to coincident (degenerate) bifurcations, as shown in Fig. 1(c). Degenerate branch points are consolidated at the geometric median of the upstream endpoint of the parent and three or more downstream endpoints of the associated children segments. In this way, two degenerate bifurcations become a trifurcation and, more generally, $n$ degenerate bifurcations become a single $(n+1)$-furcation. Networks that are hierarchically distinct in their bifurcating structure can become identical networks by collapsing bifurcations. Through exhaustive explorations (described in Sec. V A), we find that this marginally reduces the number of possible configurations. Because we have no a priori filter to identify which bifurcating trees are redundant, we must consider the entirety of the space of labeled, rooted, bifurcating trees throughout the algorithm to identify sufficiently optimal configurations. Every branch point in the network is checked and appropriately updated until the total sum of the changes in position-the Euclidean distance between the position before and after an update-is below a predetermined threshold, which we have chosen to be $0.001(<0.2 \%$ of the minimum service volume separation $\pi^{-1 / 2}$ ) in order to quickly arrive at a reasonable final configuration. With positions defined in a consistent way, we can now compare the properties of distinct hierarchies to determine which is the best for a particular space-filling strategy.

\section{SELECTION CRITERIA FOR BIOLOGICAL NETWORKS}

All characteristics of an organism that affect fitness and are heritable are under selection. A key question is which features of the vascular network are under selection. Here we define specific fitness measures that are tied to the structure of the network configuration that allows us to rank candidate networks and determine the optimal configuration.

\section{A. Global length properties of space-filling configurations}

Here we introduce a standardized fitness measure that allows us to compare candidate networks for their suitability to transport blood and resources. Each independent measure for a network's fitness relates to a physical quantity that likely guides the evolution of the cardiovascular system toward a more efficient network. Specifically, the system's cost in construction material and the maintained volume of the blood relates to the total network length - the sum of the lengths of all segments. Competing with this minimization of materials, the dissipation of the heart's pumping power relates to the path lengths between each capillary and the heart. The power dissipated by smooth (Poiseuille) flow through a segment is directly proportional to the length of the segment [24]. In the absence of radius information, reducing the cost of pumping blood is equivalent to reducing the total path lengths that blood travels.

We define these two fitness measures-one dealing with total network length and the other with individual path lengths-as

$$
\begin{gathered}
L=\sum_{\substack{\text { all segments } i \\
\text { in network }}} \ell_{i}, \\
H=\frac{1}{N_{\text {tips }}} \sum_{\begin{array}{c}
\text { all paths } p \\
\text { in network }
\end{array}} \sum_{\begin{array}{c}
\text { all segments } i \\
\text { in path } p
\end{array}} \ell_{i},
\end{gathered}
$$

where $N_{\text {tips }}$ is the number of distinct service volumes, corresponding to the number of tips and distinct paths. Total network length $L$ is addressed directly by the local positioning of unweighted Fermat points. Global information is required 
to calculate total network length, but it can be minimized if each segment length is minimized. That is, globally minimizing total path length is achievable through a sequence of local minimizations of each individual vessel length within the constraints of the optimization and network structure. Minimization of mean path length $H$ cannot be achieved via a sequence or series of local choices because whole paths (not individual segments) must be compared with one another by a search through network hierarchies. Such global information is inaccessible to a single branching junction during growth, and thus would seem to rely on information outside the realm of the actual growth process for vasculature. However, this property may not be as inaccessible as it first seems because information about mean path lengths can be partially inferred from local tissue and network properties, such as total blood flow to the area or local vessel densities, especially capillaries. The generalized total cost function $C$ linearly combines these two measures by their respective weights $C_{L}$ and $C_{H}$ :

$$
C\left(C_{L}, C_{H}\right) \equiv C_{L} L+C_{H} H .
$$

This cost function connects minimization of material and power dissipation to study optimal networks that are spacefilling.

Because an increase in cost corresponds to a decrease in fitness, we place this approach in an evolutionary framework by inverting and scaling our cost function $C$ to be a relative fitness function $f$ :

$$
f\left(C_{L}, C_{H}\right) \equiv \frac{C_{\min }}{C\left(C_{L}, C_{H}\right)},
$$

where $C_{\min }$ is the most optimal network under consideration. By this definition, the optimal configuration has a fitness $f=1$ and less optimal configurations have a fitness $f<1$.

\section{B. Equal distribution of resources through hierarchical balance}

Because the network tends to exhibit nearly symmetric branching in radius and must distribute resources equally to each capillary in the body, the network hierarchy cannot be overly unbalanced, with one segment having many more tips to supply than its sibling. In accordance with this argument, empirical data do not show major arteries branching directly into capillaries. We address this constraint by selecting for networks with more balanced hierarchies.

A hierarchy is better balanced if there are roughly equal numbers of tips supplied downstream by each sibling segment. Conversely, a hierarchy becomes more poorly balanced as the disparity grows between the number of tips. In this sense, we define the degree that a hierarchy is unbalanced $U$ as

$$
U=1-\min _{\substack{\text { all sibling } \\ \text { pairs }(i, j)}}\left\{\frac{N_{\text {tips }}^{(i)}}{N_{\text {tips }}^{(j)}}\right\},
$$

where $N_{\text {tips }}^{(i)}$ is the number of distinct downstream service volumes supplied through segment $i$ and segment $j$ is always the sibling with the most downstream tips. In our algorithm, we select against hierarchies for which the degree of unbalance $U$ is greater than some threshold $U_{0}$. This selection process is tantamount to another fitness requirement and constraint (e.g., natural selection) on the vascular network. Thus, to express all of our selection constraints within a single equation, we write down the full fitness as

$$
F\left(C_{L}, C_{H} ; U_{0}\right)=I_{U<U_{0}} f\left(C_{L}, C_{H}\right),
$$

where $I_{S}$ is the indicator (or step or Heaviside) function, which is 1 if statement $s$ is true and 0 otherwise. In our computational implementation, it is easiest to separate these steps since hierarchical balance can exclude a configuration immediately, but the calculation is exactly equivalent to the fitness function above (which may be thought of as an approximation to a more physically appropriate sigmoidal function). We eliminate configurations above this threshold before optimizing branch points and calculating fitness.

\section{GLOBAL OPTIMIZATION IN THE SPACE OF HIERARCHIES}

To determine the optimal hierarchy and its connectivity, we search the space of rooted, labeled, bifurcating trees. The positions of the branch points are fixed by the process in Sec. II C. The globally optimal network of all configurations maximizes the fitness $F$ while satisfying the space-filling constraint on service volumes. As an example, the optimal configuration in Fig. 1(d) corresponds to the hierarchy in the bottom right, where the fitness $F(1,0 ; 1)=1$ includes only total network length $L$ [Eq. (1)], resulting in a Steiner tree [25]).

Our exploration of configuration space has many similarities to phylogenetic trees, for which software is available to search through the space of hierarchies [26,27]. Since the available software is not tailored to our specific goals of optimizing space-filling networks, we implement our own algorithms. Because of the large number of distinct bifurcating rooted trees (that grows factorially with size), efficient search strategies generally focus on regions with greater fitness. We develop strategies to search through possible configurations and find space-filling networks that best satisfy the general biological constraints from Sec. III.

\section{A. Navigating in the space of hierarchies}

Our numerical technique guides the search by selecting changes that increase configuration fitness. Making small changes in the branching structure, such as a single swap of two segments in the hierarchy or a regraft of one segment to a spatially near part of the tree, yields new configurations. Because the change to the hierarchy is small, using the positions of branch points from the previous configuration as the starting positions of the iterative process described in Sec. II C saves time in optimizing the global positions in the new configuration.

For local swaps in the hierarchy, we exchange a segment with one child (including the associated downstream subtrees) of the segment's sibling (i.e., its nibling). There are $2(n-2)$ possible nibling swaps for $n \geqslant 2$ discrete service volumes. However, nibling swaps do not address changes for segments that are distant in the hierarchy but have small spatial separation. To account for these changes, we regraft single segments to spatially near branches of the hierarchy. We limit the search of spatially proximal branch points to those within twice the minimum service volume separation of each other. 
This restriction maintains a linear increase in the number of explored regrafts with the number of service volumes, in contrast to the factorial increase that would result from including all possible regrafts.

\section{B. Seed for trajectories: Balanced hierarchy construction}

We accelerate the identification of near-optimal networks by choosing an initial configuration that is maximally balanced (i.e., minimizes $U$ ) and avoids many suboptimal structures (e.g., configurations with many repeated crossings or noncontiguous subtrees) for every set of service volume distributions. To improve overall computation time, some approaches explore permutations of pairing tips with service volumes under a constant hierarchy [28]. For real systems, the dimensionality of the space (i.e., the body volume) for each branch point position never exceeds three, which allows us to use spatial partitioning to directly construct a configuration, with compact, nonoverlapping subtrees. Such a favorable configuration avoids less-fit configurations and satisfies the intuitive guidelines that branch points connect nearby subtrees (efficiency by proximity) and that sibling subtrees have similar numbers of service volumes in accordance with symmetric branching in radius.

To ensure the maximal hierarchical balance for the seed, we begin with a single set that contains all terminal service volumes. This set is then partitioned into two subsets of equal size (or within 1 service volume if the number is odd, which guarantees $U \leqslant 0.5$ ), using a straight line to define the boundary between the two sets. When appropriate, this line passes through the geometric center (i.e., the unweighted average position) of the previous set of points and the geometric center of the new subset. Resulting sets further split into smaller subsets to yield a complete, bifurcating hierarchy. We refer to this process and the resulting seed as the balanced hierarchy construction (BHC).

\section{Efficient search trajectories}

We further accelerate our search by limiting the number of nearby configurations considered at each step. We accomplish this through a carefully guided greedy search through the space of hierarchies (effectively simulated annealing [29,30] at zero temperature), which often finds a near-optimal configuration. A greedy strategy offers expedited elimination of configurations that are far from optimal; the algorithm abandons configurations that saturate at a fitness lower than the current most optimal configuration. Our implementation allows five iterations of the process in Sec. II C, then excludes configurations that fail to reduce the $\operatorname{cost} C[\mathrm{Eq}$. (3)] by at least $5 \%$ of the remaining difference from the current optimal configuration. The algorithm with this exclusion scheme successfully identifies near-optimal configurations.

Because the sampling process is not exhaustive, the search through the space of possible hierarchies is not guaranteed to yield a globally optimal configuration. However, performing a reasonably thorough search as we outline here and conducting several runs from the BHC seed (in our simulations, at least ten runs) increase the likelihood of identifying a configuration that is near-optimal and shares many of the branching length asymmetries that an optimal configuration exhibits. With dependable algorithms for determining branch point positions and for exploring the space of possible hierarchies, we can now investigate the length properties of space-filling networks under several basic space-filling strategies.

\section{RESULTS AND ANALYSIS}

We now present the results of optimized networks and of the analysis on real vascular networks, including the properties of the most optimal networks. To build intuition about the space of hierarchies, we first explore the space exhaustively for small networks and establish the distinct patterns that the two optimizations $L$ and $H$ produce. In comparing optimal configurations with observations of real systems, we find better agreement by enforcing a constraint on the degree of unbalance $U$ in the hierarchies of candidate configurations.

\section{A. Exhaustive search for small networks}

To become more familiar with the landscape of possible configurations, we exhaustively explore the space of hierarchies and pairings for networks that are small enough to quickly yield comprehensive results for a single realization of fixed service volumes. We collapse and reorganize the higher-dimensional space of branch point swaps into a single dimension by ranking each configuration based on the fitness $F$. This reorganization involves a normalization of rank so that the fittest configuration occurs at 0 and the least fit occurs at 1 . Rescaling the rank is necessary even for networks of the same size and shape because different realizations may have different numbers of unique configurations after consolidating degenerate bifurcations (mentioned in Sec. II C), despite having the same number of service volumes. Larger networks tend to have greater range with respect to both costs in $L$ and $H$.

The minimum distance between each service volume is constant for each of the networks that constitute the ensemble of realizations for the curves in Fig. 2. Each curve represents the average fitness (relative to the fittest configuration for the particular set of capillary positions, represented by the subscript best) over an ensemble of networks with a fixed number of tips and constant total area. In generating the ensemble, we exclude those that arise with a different number of tips than what is desired until we accumulate 1000 configurations of the target size. Across curves, the total area increases to produce networks with more service volumes more frequently.

One might expect a large set of similarly fit, near-optimal networks, which would be represented by a plateau near the optimum. However, the sharp descents away from the optimal configuration in Fig. 2 indicate that there are few configurations that are near-optimal. From an evolutionary perspective, this implies that the vascular networks of organisms are under strong selection. Furthermore, the slope near the optimum becomes steeper as more service volumes are introduced, so that the best configurations become more distinct from other possibilities as the number of service volumes grows. Considering the very large number of service volumes in real organisms, this again indicates that real vascular networks 

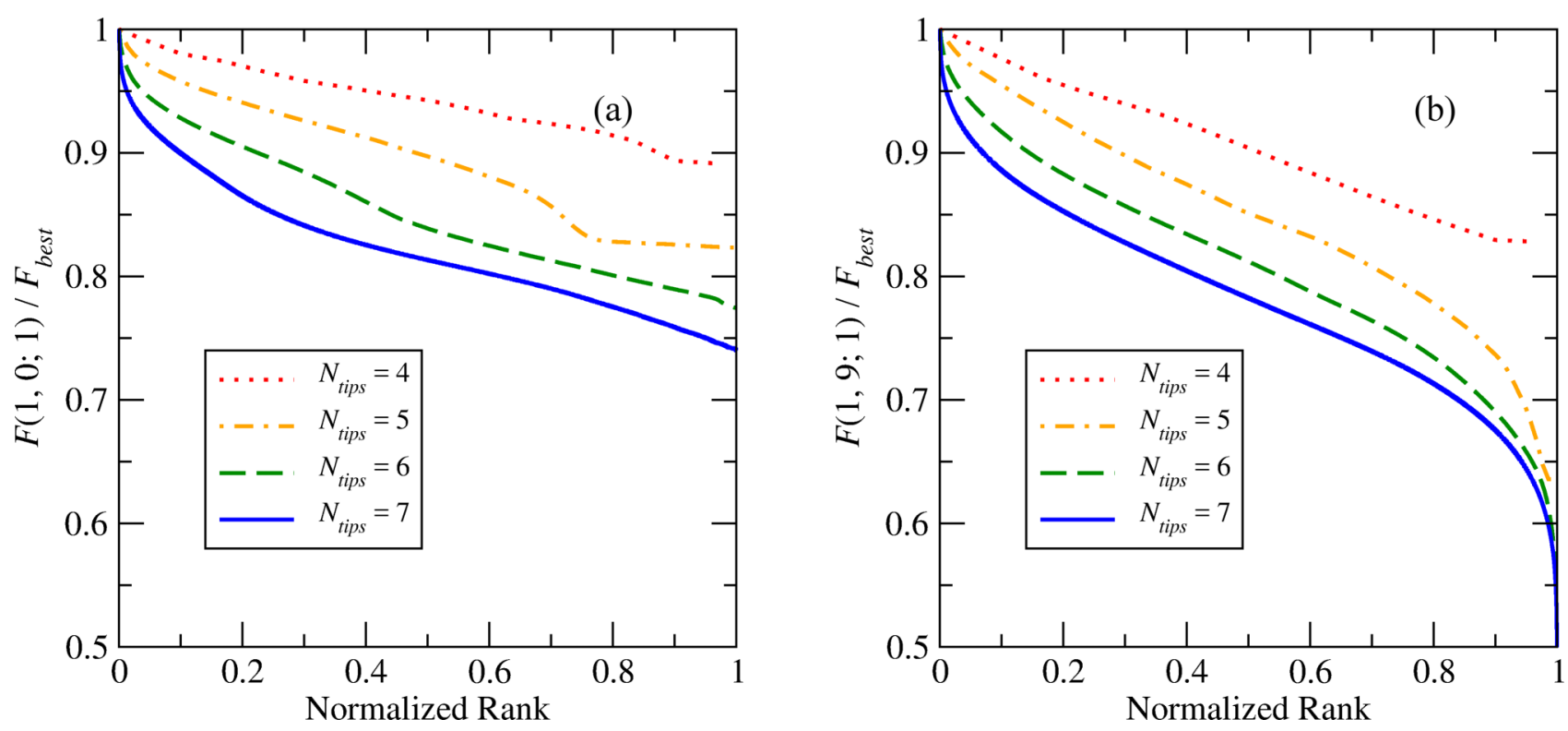

FIG. 2. Ranked landscapes for unique consolidated configurations from exhaustive exploration of bifurcating trees of fixed area and fixed $N_{\text {tips. }}$. (a) Exhaustive landscapes for fitness based only on total network length $L[F(1,0 ; 1)]$. (b) Exhaustive landscapes for fitness based total network length $L$ and mean path length $H[F(1,9 ; 1)]$. We choose the weights $\left(C_{L}, C_{H}\right)=(1,9)$ so that the contribution from $H$ is not dominated by the contribution from $L$.

are under strong selection pressures for space-filling and efficiency.

Optimal networks that have no constraint on hierarchical balance fall into two general classifications depending on the relative weights of total network length $L$ and mean path length $H$ in the fitness measure $f$. As shown in the simple examples of Fig. 3, network fitness measures that are weighted to minimize $L$ yield bifurcating trees, while measures that are weighted to minimize $H$ yield hubs. Bifurcating trees better correspond to real networks, suggesting that total network length $L$ plays a larger role than mean path length $H$. Since a single hub is not observed (and not expected from material costs) in real systems, we do not consider configurations that ignore total
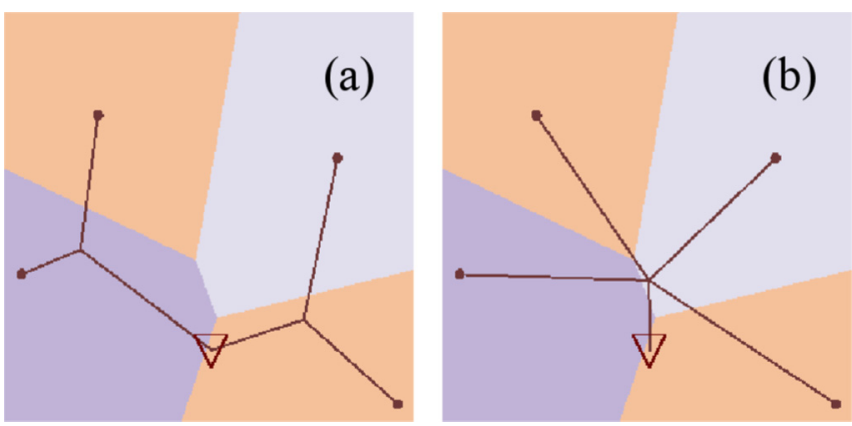

FIG. 3. Global optimizations in the space of configurations for two classes of networks: (a) The optimal configuration that minimizes total network length $[L$ in Eq. (1) for $F(1,0 ; 1)]$ of the 15 possible trees [corresponding to Fig. 1(d)] consists only of bifurcations. (b) The optimal configuration that minimizes mean path length between each service volume and the heart $[H$ from Eq. (2) for $F(0,1 ; 1)]$ of the 15 possible trees consists of a single hub. The regions of varying background color define the Voronoi cells corresponding to individual service volumes. network length $L$. Therefore, the hub in Fig. 3(b) occurs at the geometric median of the branch point's adjacent segment endpoints in accordance with the method presented in Sec. II C instead of at the trivial minimum of path lengths in which each service volume has a unique segment that connects it directly to the heart. However, optimizing only for $L$ leads to meandering, bifurcating paths, which become shorter and more direct by including both costs ( $L$ and $H$ ). Furthermore, more global information is necessary to directly minimize path lengths than the local environment that we consider in Sec. II Cspecifically, the context of the entire path. This means that our analysis is best suited for optimality that always includes a significant contribution from total network length $L$ and a weaker contribution from mean path length $H$.

\section{B. Trajectories for sampling larger networks}

With better intuition about the space of hierarchies from small networks, we now explore the space for larger networks with more service volumes. Branching asymmetries have been corroborated in small distribution networks from a central source to an outer boundary [31]. Here we present results for larger networks with the perimeter and the entirety of the inner space filled with tips. The branching properties of larger networks give more applicable results to connect particular space-filling strategies with the observations of real cardiovascular systems. We first summarize the properties of optimized networks without any constraint on hierarchical balance $\left(U_{0}=1\right)$.

Because the search through the space of hierarchies is not exhaustive for large networks, we cannot show ranked landscapes averaged over ensembles with different service volume positions as we did for small networks. Instead, we show landscapes from a single realization of service volume positions in Fig. 4 that come from an ensemble of trajectories that start with 


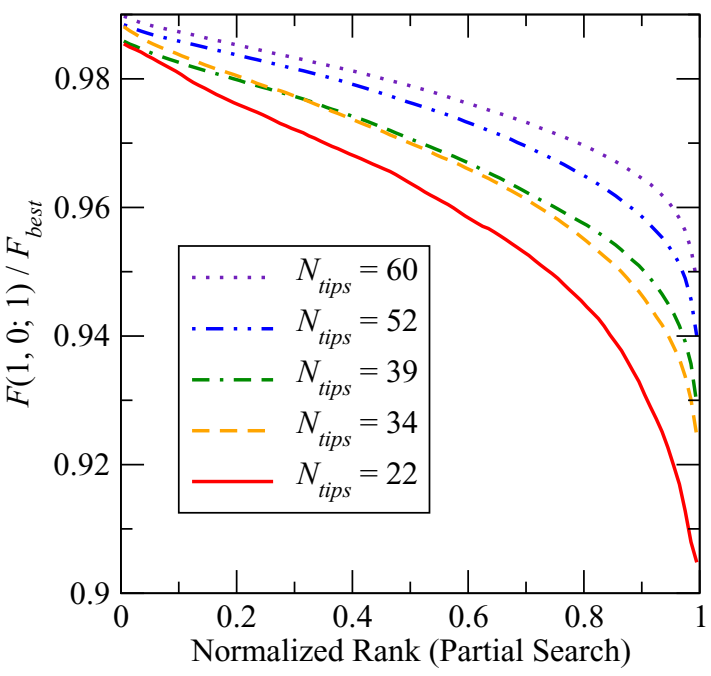

FIG. 4. Average fitness landscapes for total network length $L[F(1,0 ; 1)]$ over 100 trajectories for a single network of each size.

the BHC configuration and end at a local optimum [Fig. 11(a) in Appendix A]. The greedy algorithm samples fewer less-fit configurations, yielding a shallower slope near the optimum than the exhaustively explored landscapes in Fig. 2. Since the starting point of the search (the BHC configuration) is already favorable, we expect that the worst-ranked configuration of the partial search is already very near optimal. While this may not be the absolutely optimal configuration, we do not expect the absolute optimum to be found by real biological networks either. This is both because additional constraints will likely influence network growth and because there is noise in any growth process that is this complex. However, real networks and the best artificial configurations should both be near optimal. Indeed, for fitness landscapes that we can explore exhaustively (as shown in Fig. 13(b) in Appendix B), we show that the optimal network is found in the majority of searches. For cases where the absolute optimal configuration is not correctly identified, the algorithm finds a nearly optimal configuration. As the number of tips increases, there is a decreasing difference between the absolute optimal network and the nearly optimal network identified (average normalized ranked) by our algorithm. This result suggests that there will be negligible qualitative differences between the network found by our algorithm and the absolute optimal network in the context of real biological networks, which are much larger. We provide further details on the performance of this partial sampling of the configuration space in Appendix B.

Searches through the space of hierarchies and the properties of optimal configurations do not vary with different convex body shapes. Figure 5 shows examples of optimized networks for a maximally symmetric body shape. The general trends of long, meandering paths for solely minimizing total network length $L$ and of more direct paths when including $H$ are consistent across both isotropic, circular areas and elongated, rectangular areas.

To characterize branching features of both our empirical data and these large configurations, we quantify the asymmetric branching attributes with the two ratios

$$
\begin{aligned}
\lambda_{L} & =\frac{\ell_{c_{1}}}{\ell_{c_{2}}}, \\
\lambda_{R} & =\frac{r_{c_{1}}}{r_{c_{2}}},
\end{aligned}
$$

choosing $\ell_{c_{1}} \leqslant \ell_{c_{2}}$ for the lengths of child 1 and 2 [shown in Fig. 1(a)] and $r_{c_{1}} \leqslant r_{c_{2}}$ for the data on radii. Note that perfect symmetry corresponds to $\lambda_{L}=\lambda_{R}=1$ and smaller values of $\lambda_{L}$ and $\lambda_{R}$ correspond to more asymmetric branching. Distributions for the branching asymmetry ratio in length $\lambda_{L}$ for various sizes and shapes are shown in Fig. 6. Branch points for which one of the child segments does not exist because of degeneracy with a service volume center do not contribute to the distribution for $\lambda_{L}$. There is little change in the features of the distribution of $\lambda_{L}$ across different sizes of networks with $U_{0}=1$. This trend persists for both isotropic and anisotropic enclosing shapes. A summary for the cross-generational length ratio is given in Appendix C.

Many branch points in these networks coincide with a service volume, predicting large trunks that feed capillaries directly. Similar results appear in the study of flow through a dynamic, adaptive network [32]. However, such a trend does not agree with the empirical data. Although there is asymmetry
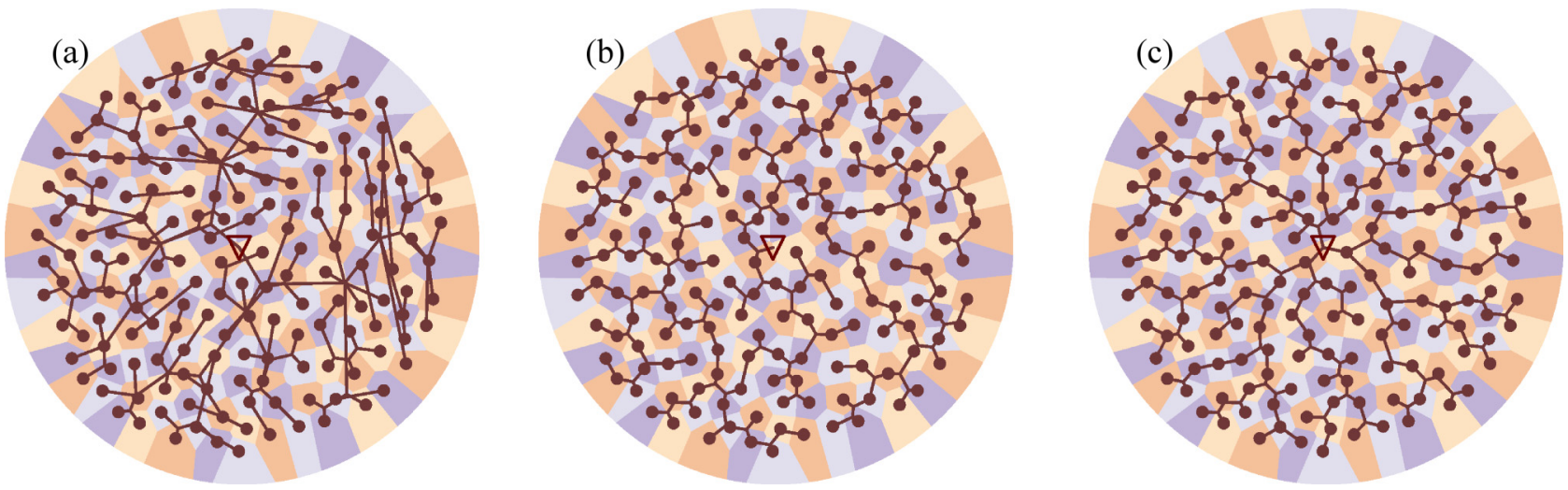

FIG. 5. Network configuration for (a) the BHC seed and two optimal configurations for fitness measures (b) total network length $L[F(1,0 ; 1)]$ and (c) total network length $L$ and mean path length $H[F(1,9 ; 1)]$. We choose the weights $\left(C_{L}, C_{H}\right)=(1,9)$ so that the contribution from $H$ is not dominated by the contribution from $L$. 


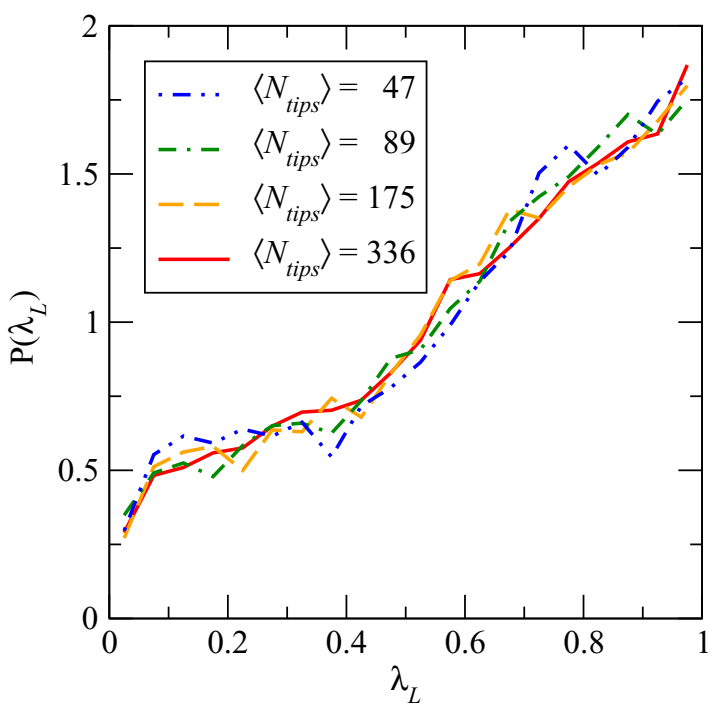

FIG. 6. Distributions of $\lambda_{L}$ for several sizes of circular areas, averaged over 100 realizations of service volume distributions and optimized solely for total network length $L[F(1,0 ; 1)]$. Local length asymmetry between siblings skews toward symmetry for optimal networks with little hierarchical balance $\left(U_{0} \approx 1\right)$.

in adjacent segments at branch points and a lack of strict balance in the hierarchy along different paths, we observe that large arteries do not branch directly to capillaries and arrive at the same expectation from the dynamics of blood flow. The major qualitative distinction between the BHC and the optimized configurations with $U_{0}=1$ is that the $\mathrm{BHC}$ is a network with a balanced hierarchy. Upon inspection of empirical data in Sec. V C, we find that the branching length asymmetries for the $\mathrm{BHC}$ configuration motivate an additional constraint on hierarchical balance during the search through the space of hierarchies.

\section{Comparison of optimized networks with empirical data}

The results in Sec. V B show that optimization for total network length or mean path length with no constraint on hierarchical balance leads to distributions of asymmetry in sibling vessel length that skew toward symmetry $\left(\lambda_{L} \approx 1\right)$. We now present the analysis of $\lambda_{L}$ that characterizes the local length asymmetries at branch points for real and optimized networks. From this analysis, we explore how limiting the degree of unbalance $U$ in an optimal artificial network yields asymmetries that better match biological networks.

\section{Asymmetric vessel length distributions of real networks}

We analyze MRI images of the human head and torso as well as microtomography images from wild-type mouse lung. Both data sets break from strict symmetry. As shown in Fig. 7, the network-wide distribution for $\lambda_{R}$ is skewed toward symmetry $\left(\lambda_{R} \approx 1\right)$, while the distribution for $\lambda_{L}$ is more uniform, representing a greater contribution from very asymmetric branching $\left(\lambda_{L}<1\right)$. These results are representative of general features for length distributions in real biological networks. The fact that the optimized networks in Sec. V B do not exhibit a similar distribution for $\lambda_{L}$ signals that important biological factors are missing. Because of the skew toward symmetry in sibling segment radii, we limit the hierarchical unbalance of optimized networks in Sec. V C 2.

\section{Degree of balance necessary to match biological networks}

Imposing a constraint on hierarchical balance leads to configurations that reflect more realistic asymmetry in branching lengths. Hierarchical balance, which equalizes the number of service volumes that each sibling segment supplies, is related
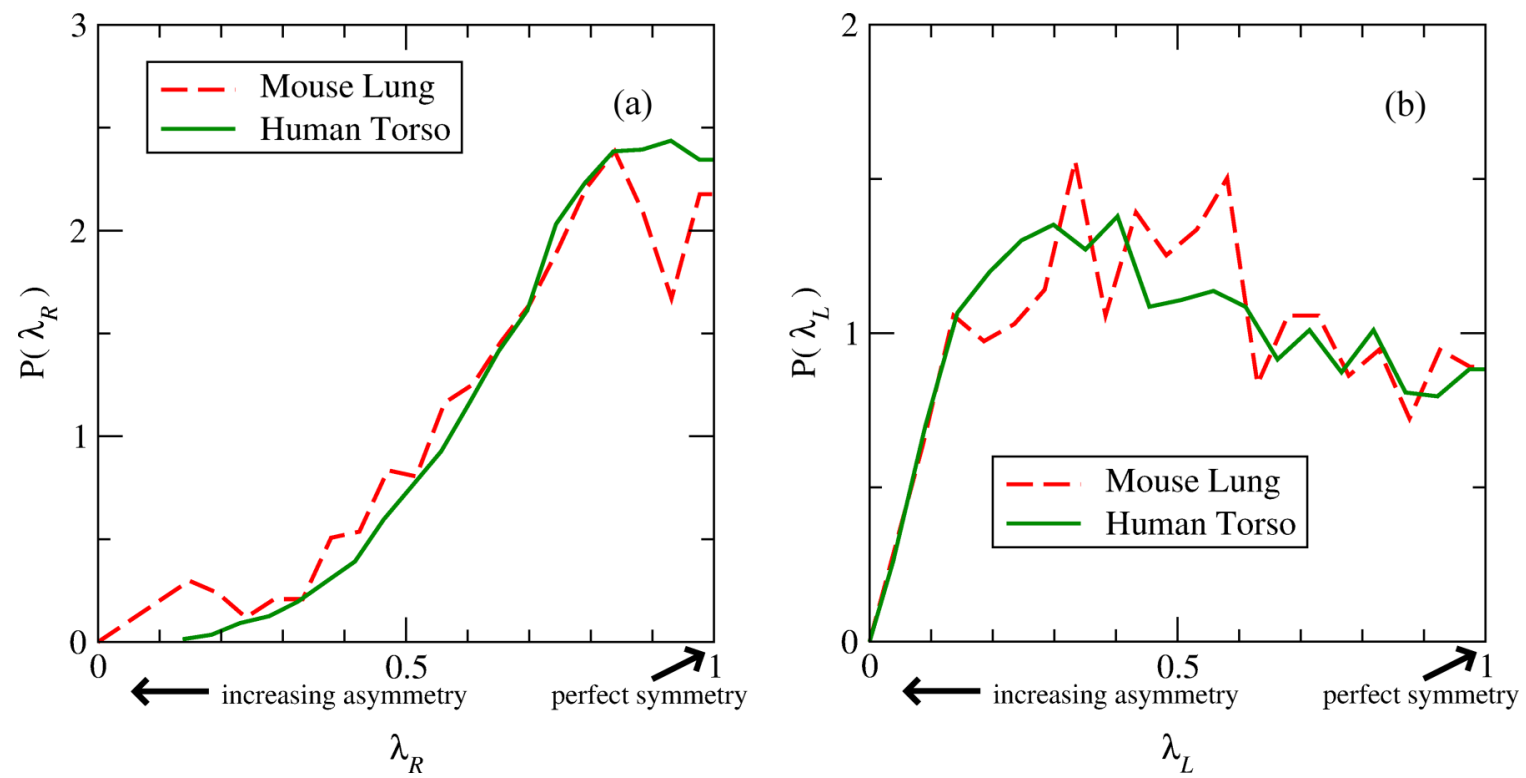

FIG. 7. Observed radius and length branching asymmetry ratios [Eqs. (7) and (8), respectively] in mouse lung and human torsos. (a) Sibling radius ratio $\lambda_{R}$ distribution. Radii ratios are skewed toward symmetry $\left(\lambda_{R} \approx 1\right)$, although they are not always perfectly symmetric. (b) Sibling length ratio $\lambda_{L}$ distribution. Length ratios are not skewed toward symmetry (many ratios have $\lambda_{L}<1$ ), contrary to symmetric models. 

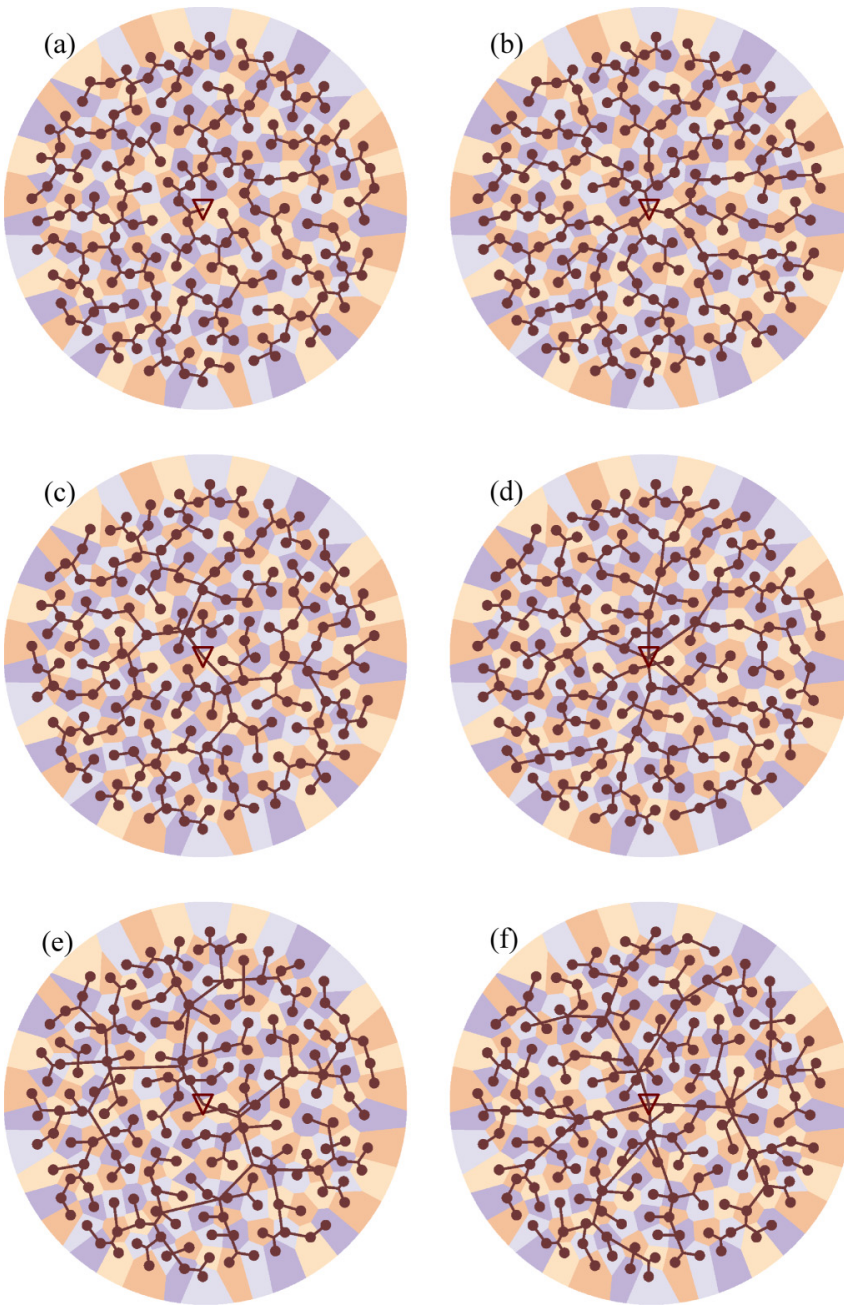

FIG. 8. Optimal configurations for several constraints on hierarchical balance and two optimization weights for fitness $F$. The left column (a, c, e) shows configurations optimized for total network length $L\left[F\left(1,0 ; U_{0}\right)\right]$, while the right column (b, d, f) shows configurations optimized for both total network length $L$ and mean path length $H\left[F\left(1,9 ; U_{0}\right)\right]$. The rows from top to bottom show configurations for $U_{0}=1.0,0.9$, and 0.6. The BHC seed is the same as in Fig. 5. We choose the weights $\left(C_{L}, C_{H}\right)=(1,9)$ so that the contribution from $H$ is not dominated by the contribution from $L$.

to the blood flow that is required to deliver resources and effectively limits the asymmetry of sibling radii. In Fig. 8 we show results for several thresholds for the constraint on hierarchical balance. Decreasing the threshold $U_{0}$ yields more realistic distributions for $\lambda_{L}$, as shown in Fig. 9. Because these networks are embedded in $2 \mathrm{D}$, decreasing $U_{0}$ can also result in more crossings between segments at different levels. By comparing Figs. 9(a) and 9(b), we see that the constraint on hierarchical balance leads to similar results independent of the weight of mean path length $H$ in configuration fitness. Instead of contributing significantly to fitness, $H$ is effectively optimized through hierarchical balance.

While enforcing hierarchical balance leads to more realistic branching and length asymmetry distributions, it is not necessary to have a maximally balanced hierarchy. In Fig. 10, we show that lowering the threshold $U_{0}$ reduces network fitness.
As the threshold for hierarchical unbalance $U_{0}$ decreases, the average fitness also decreases. Since the distribution of $\lambda_{L}$ is approximately uniform around $U_{0} \approx 0.7$ and below, the best value for the hierarchical constraint is $U_{0} \approx 0.7$, because this yields the fittest networks that have uniform distributions for length asymmetry.

\section{DISCUSSION}

With our determination of branch point positions and exploration of distinct hierarchical configurations, we can remark on several consequences that follow from the general properties of optimized networks. Organizing the lengths between branch points to fill 2D or 3D space with capillaries inevitably leads to asymmetries and unbalanced networks [33]. Strictly symmetric and balanced networks are either inefficient in materials or not space-filling. For example, in the H-tree all children branch orthogonally from the parent, resulting in inefficient paths. Other networks with more efficient paths lead to capillaries that are equidistant from the source, which could cover the surface of a sphere but not fill its volume. For the optimal, space-filling networks that we explore, we impose a constraint that pushes the network toward hierarchically balanced branching structures but does not require maximum balance. One can imagine other interesting metrics for hierarchical balance, but we concentrate on how a maximum degree of unbalance $U_{0}$ affects the structure of the network. This guarantees a minimum level of balance in the hierarchy but still allows freedom in the search for optimal networks, as well as nonuniformity in the hierarchical balance.

We construct a seed configuration that builds a network to ensure maximal hierarchical balance while maintaining efficient contiguity of subtrees. Configurations that tend to be hierarchically balanced, such as the BHC configuration (where the constraint is implicit in the construction algorithm) or optimized configurations that limit unbalance, do not show a strong skew toward symmetric branching in lengths. This hierarchical balance may result from gradual, incremental growth as an individual organism matures and ages. Nearby vessels grow to supply resources to new tissue, resulting in contiguous subtrees and favoring routes that reduce path lengths and avoid a single, meandering artery that branches directly to capillaries.

Other computational models approach the growth and optimization of space-filling networks in different ways. Although there are many algorithms to generate structure that do not intentionally optimize network architecture or spacefilling properties, near-optimal configurations may emerge spontaneously from certain simple rules. Examples of such pattern formation processes and associated algorithmic rules include models for both angiogenesis [19,34], as well as vasculogenesis (in terms of chemotaxic [35,36], mechanical substratum [37], and cellular Potts models [38,39]). However, these models do not adequately address our focus on branching-length asymmetries for efficient, hierarchical, space-filling networks. Specifically, the pattern formation model for angiogenesis does not incorporate consistent spacefilling service volumes, only space-filling arterial structure. The arterial structure fills some regions so that they are devoid of capillaries, while multiple tips converge to the same location 

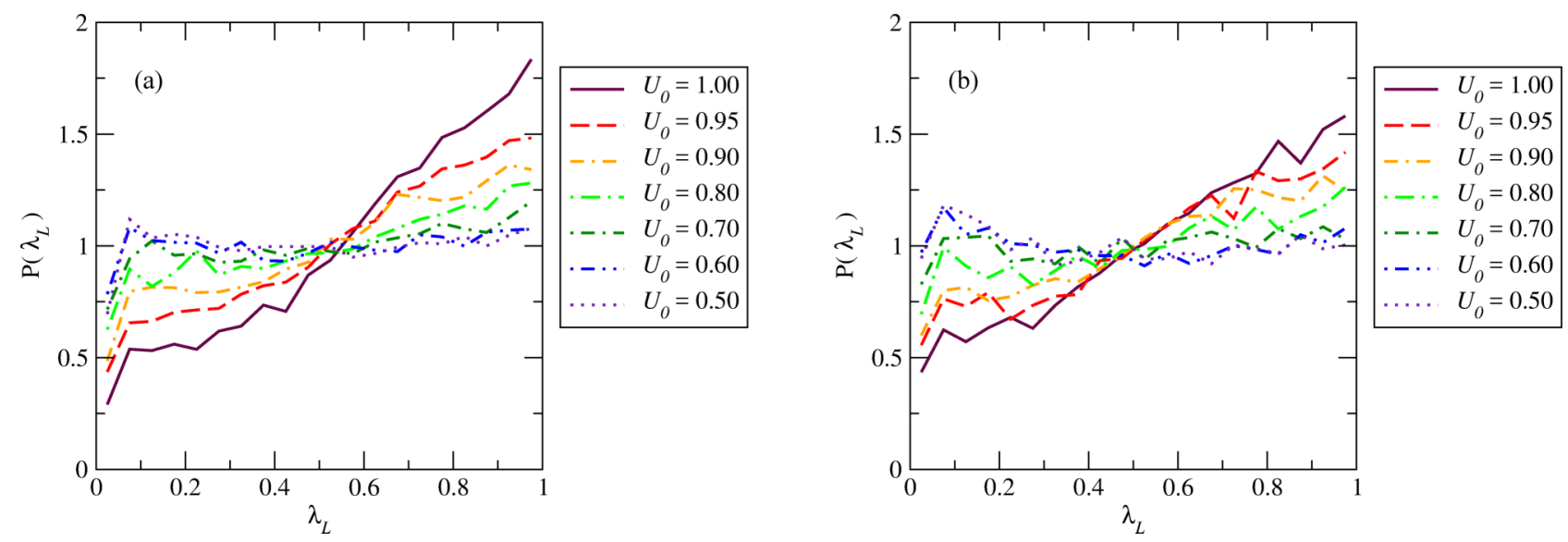

FIG. 9. Distributions for several thresholds of hierarchical unbalance $U_{0}$. (a) Distributions of $\lambda_{L}$ optimized solely for total network length $L\left[F\left(1,0 ; U_{0}\right)\right]$. (b) Distributions for $\lambda_{L}$ optimized for total network length $L$ and mean path length $H\left[F\left(1,9 ; U_{0}\right)\right]$. All plots are averaged over 200 realizations of service volume distributions.

elsewhere. The models for vasculogenesis do not optimize the development of a hierarchical branching network. However, dynamic vascular remodeling [32] can form structures both with and without closed loops while maintaining a uniform distribution of capillaries, although the optimal structures also suffer from large arteries branching directly to capillaries. Recently, an alternative search strategy, simulated annealing, has been applied directly for the positioning of branch point locations utilizing the properties of blood flow to fill a space with tips by Ref. [40]. In order to cover larger ensembles of networks and devote more computational resources to exploring different hierarchies, we specify the optimal location for branch points given a specific hierarchy, which removes the need to search through the possible locations of points through simulated annealing. We extend and standardize these models to understand the asymmetric lengths of adjacent segments in

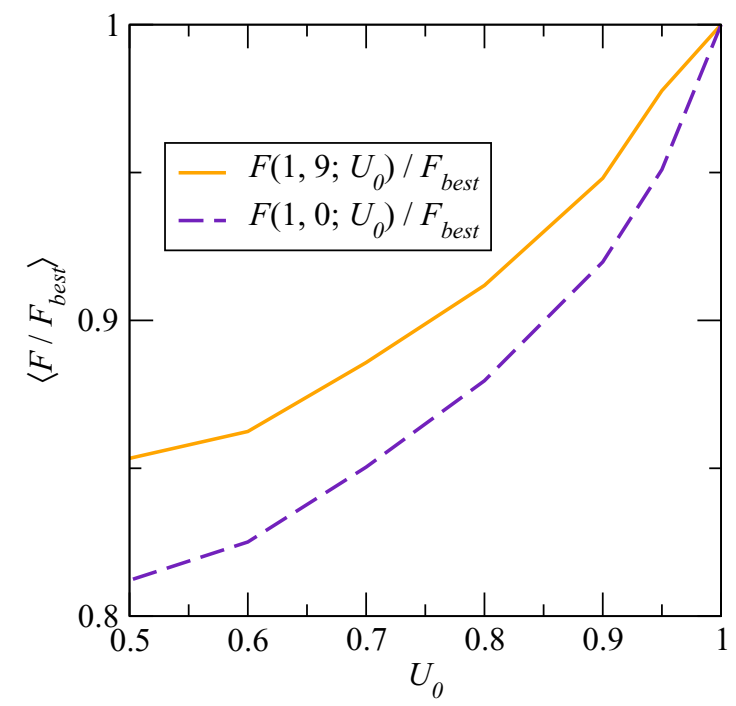

FIG. 10. Average fitnesses $F$ for ensembles of 100 optimized configurations. Configurations are more fit if a greater hierarchical unbalance $U_{0}$ is allowed, although the distribution for $\lambda_{L}$ does not change much beyond $U_{0} \approx 0.7$ (see Fig. 9). vascular networks and how these relate to space-filling service volumes.

Because of the many different factors and interactions that influence the structure of the cardiovascular system, our basic model can be expanded in several directions. Radius information can be incorporated into optimized networks by requiring flow to be uniform in all terminal service volumes. By including radius information, blood flow as well as more appropriate structural and energetic costs can lead to revised optimization principles, which require the calculation of the weighted Fermat point (see, e.g., Ref. [21]) and has been explored previously in a limited, local context [24,41,42]. Note that lowering the threshold $U_{0}$ tends to increase the minimum number of branching levels between the heart and capillaries. Less drastic hierarchical unbalance implies that the ratios of parent-child radii $\beta=r_{c} / r_{p}$ should be near 1 (symmetric branching). This translates the global, topological property into a local branching quantity.

Increasing the dimensionality of our networks to 3D does not change the qualitative results for branching asymmetry in length $\left(\lambda_{L}\right)$ with hierarchical balance (specifically $\left.U_{0}\right)$. The exact location of the optimal tradeoff between fitness and balance does shift, but only slightly. The details in the quantitative shift of the tradeoff, as well as the role that different forms of the hierarchical unbalance may play could both be explored for $3 \mathrm{D}$ in the future. Studies of large vessels (near the heart) show the branching of these vessels to be planar [43], but the planarity cannot always hold across the entire network if tips must fill a 3D space. Nevertheless, in the absence of obstacles, all optimization conditions enforce planarity in 3D for branch points in their local context. Introducing regions where the network is prohibited (e.g., through bones, organs, or from self-avoidance) constrains the Fermat point to the surface of a sphere or some other shape $[44,45]$.

While the topological change of allowing loops introduces many complications to the properties of flow and hierarchical labels [46], such a modification can be beneficial in understanding reticulated vascular structures. Loops are especially important when considering network robustness (i.e., resilience to damage) within organs and leaves $[16,17]$ or 

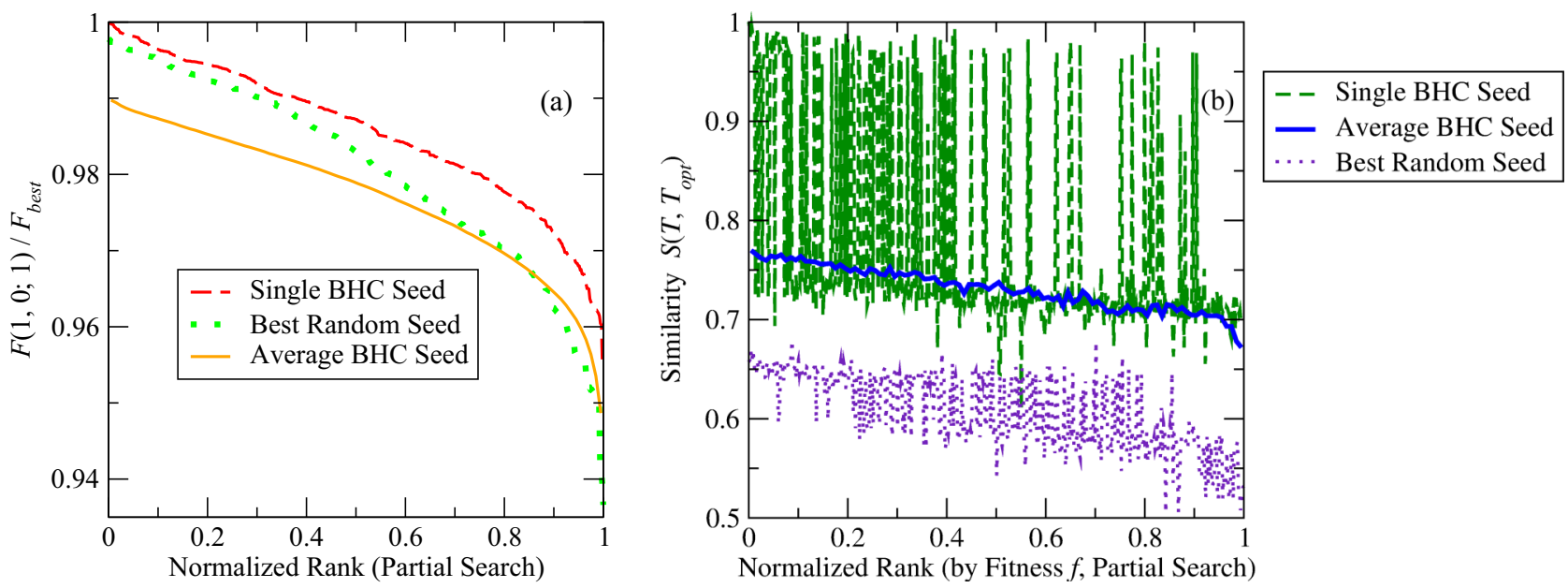

FIG. 11. Partial search trajectories in the the space of hierarchies. (a) Ranked fitness landscapes for the partially explored hierarchical space during optimization for total network length $L[F(1,0 ; 1)]$. (b) Corresponding similarity measures. All quantities are calculated with respect to the most optimal configuration found over all runs. The solid line shows the average fitness over 100 trajectories from the BHC for a system with 36 service volumes. Individual paths are for a network of 48 service volumes. One path begins with the BHC configuration and the other begins with a random seed (the best performing of 5 random seeds).

pathological growth in tumors $[47,48]$. These types of network properties can be included in future models.

Locally, the position of a branching junction minimizes the sum of vessel lengths in our model. Globally, we impose a threshold on the minimum hierarchical balance, which reduces the differential blood flow into sibling segments. Although real vascular networks consist almost entirely of bifurcations (although there is rapid, asymmetric branching from the aorta to capillaries through coronary arteries), the iterative approach described in Sec. IIC can lead to low numbers of bifurcating junctions for some candidate networks.

Limiting the degree of unbalance in the hierarchy does not continue to shift the distribution of $\lambda_{L}$ away from symmetry $\left(\lambda_{L} \approx 1\right)$ below $U_{0} \approx 0.7$, which suggests that there is an appropriate tradeoff between the hierarchical unbalance threshold $U_{0}$ and configuration fitness $f$ that does not require perfect symmetry for an efficient network structure. The increased cost of the network in Fig. 10 is similar for both curves, implying that the increase mostly comes from total network length $L$.

The large number of distinct bifurcating hierarchies necessitates that we carefully choose and execute the algorithms for searching the space of possible configurations. Consequently, we construct a favorable starting point and concentrate computational resources on regions that are most likely to contain optimal configurations. Using the numerical implementations
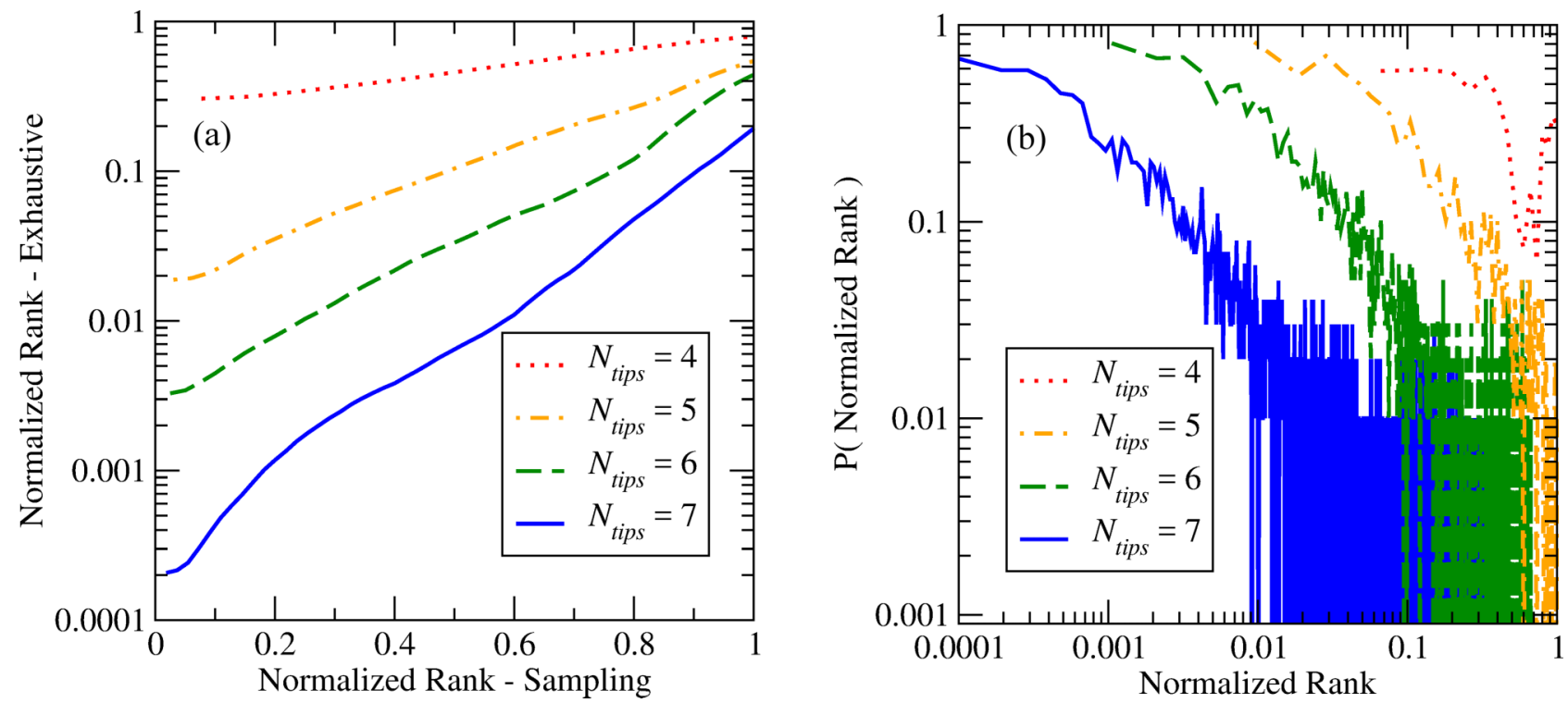

FIG. 12. Average sampling of the partial search algorithm over an ensemble of 100 realizations of tip positions for networks that are small enough to explore the space of configurations exhaustively. Note the log ordinates. (a) The average rescaled exhaustive rank improves exponentially as new configurations are explored during sampling. (b) The probability of sampling a given exhaustive rank during a partial search of the space of hierarchies shows that few poor configurations are sampled, while the fitter configurations are sampled heavily. 
in Secs. II and IV, we identify optimal networks and study the length properties of individual segments within the context of a network with space-filling terminal service volumes.

Our results have many implications for how vascular networks fill space efficiently. We exhaustively explore fitness landscapes for small networks and carefully guide the sampling of the space of hierarchies for large networks in order to determine near-optimal configurations. Our results show that strict hierarchical balance is not optimal for the architecture of cardiovascular networks. Furthermore, there is a tradeoff between hierarchical balance (which is related to symmetric branching in radius at the local level) and the distribution for branching in lengths that shows the connection between the space-filling and efficiency requirements of the network. By incorporating radius and flow information, as well as growth patterns that incorporate obstacles and loops, we can continue to build on present models to better understand vascular architecture and gain insights for its effects on resource delivery, metabolic scaling, aging, and repair after damage.

\section{ACKNOWLEDGMENTS}

We thank Kristina I. Boström, MD, Ph.D., and Yucheng Yao, MD, Ph.D., for sharing their data of mouse lung vasculature. We also thank Daniel Ennis, Ph.D., for sharing his data of human head and torso vasculature and Mitchell Newberry for his work in developing the software to analyze vascular images. We are grateful to Eric Deeds, Ph.D., and Tom Kolokotrones, MD, MPH, Ph.D., for engaging in stimulating discussions about the work. We also thank Elif Tekin for her patient help in refining the presentation in this article. D.H. and V.M.S. received funding from the U.S. National Science Foundation (Grant No. 1254159).

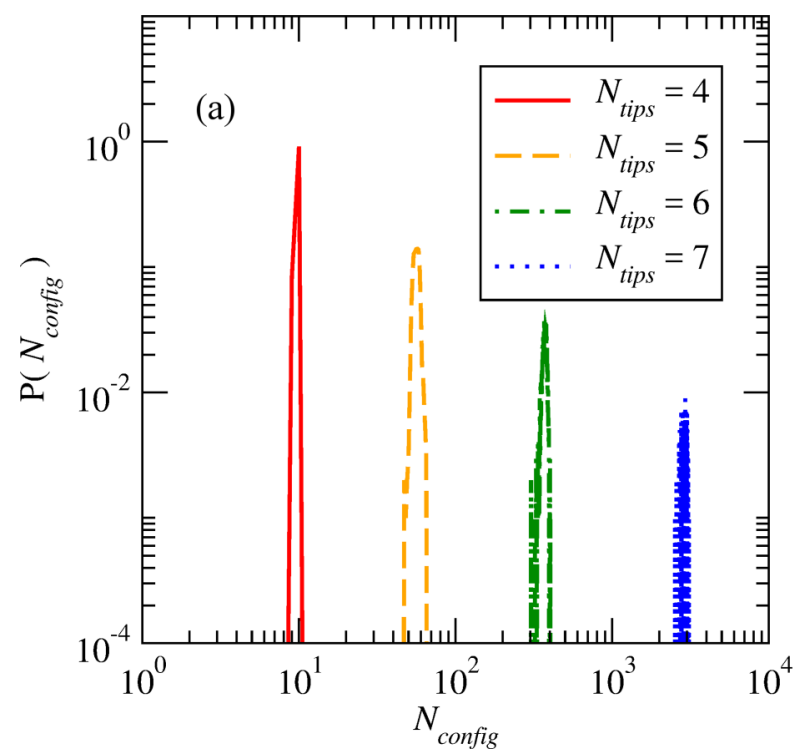

\section{APPENDIX A: SIMILARITY MEASURE TO COMPARE HIERARCHICAL GROUPINGS BETWEEN CONFIGURATIONS}

While collapsing the landscape of measures to a single dimension informs us about the typical distribution of configurations, it retains no information about the relation of the hierarchies between different trees. To address this issue, we define a measure of similarity to compare how two hierarchies group the same set of tips. This measure is normalized such that similar hierarchies and groupings of service volumes have a similarity score near 1 , while hierarchies that group service volumes in very different ways have a similarity score near 0 . To meet these guidelines, we perform a simple count of the number of identical subtree groupings between two hierarchies and normalize by the maximum possible number that could be shared if the trees were identical. In accordance with these properties, define the similarity $S(A, B)$ between two configurations $A$ and $B$ as

$$
\begin{aligned}
S(A, B) & \equiv \frac{\sigma(A, B)}{\max \{\sigma(A, A), \sigma(B, B)\}}, \\
\sigma(X, Y) & =\sum_{\substack{\text { subtree } m \\
\text { in network } X}} \sum_{\begin{array}{c}
\text { subtree } n \\
\text { in network } Y
\end{array}}\left(I_{m \subseteq n}+I_{n \subseteq m}\right),
\end{aligned}
$$

where $I_{S}$ is the indicator function (1 if statement $s$ is true and 0 otherwise) and subtree refers to the set of tips in that particular subtree.

Configurations that have a worse fitness measure are less similar to the optimal configuration, as shown in Fig. 11.

However, note that similarity $S\left(T_{i}, T_{\text {opt }}\right)$ is not a monotonic function when rank $i$ is defined by the configuration's measure. For example, consider hierarchy A, which may be very

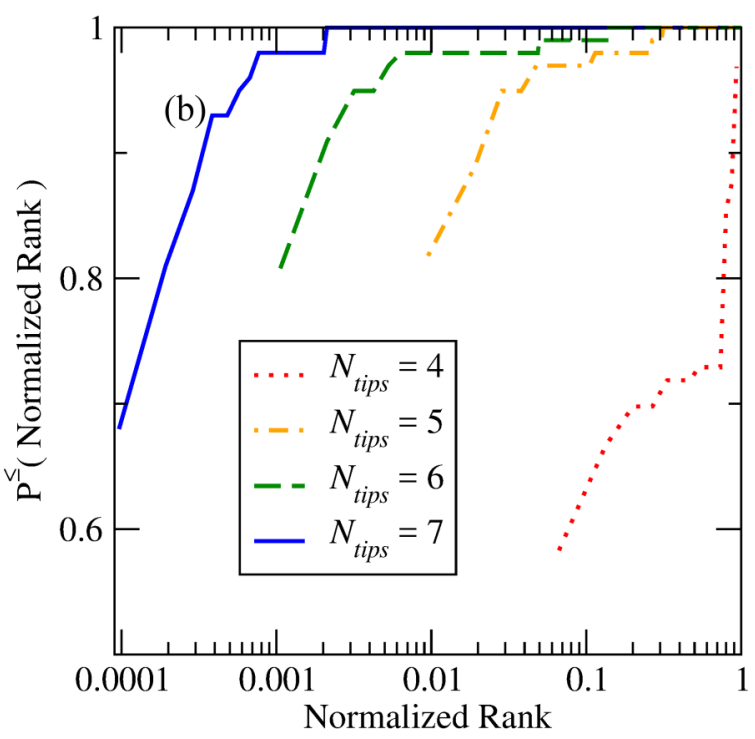

FIG. 13. (a) The number of unique configurations $N_{\text {config }}$ for each fixed number of service volumes is narrowly distributed relative to the increased number of configurations from introducing an additional service volume. (b) The average probability (over 100 realizations of tip positions) that a configuration at least as fit as the given rank's configuration is found (i.e., the probability $P \leqslant$ that a rank less than or equal to the given rank is found) during a sampling through configurations. The search correctly identifies the optimal configuration in most attempts, and finds very near-optimal configurations otherwise. Note the log abscissa. 

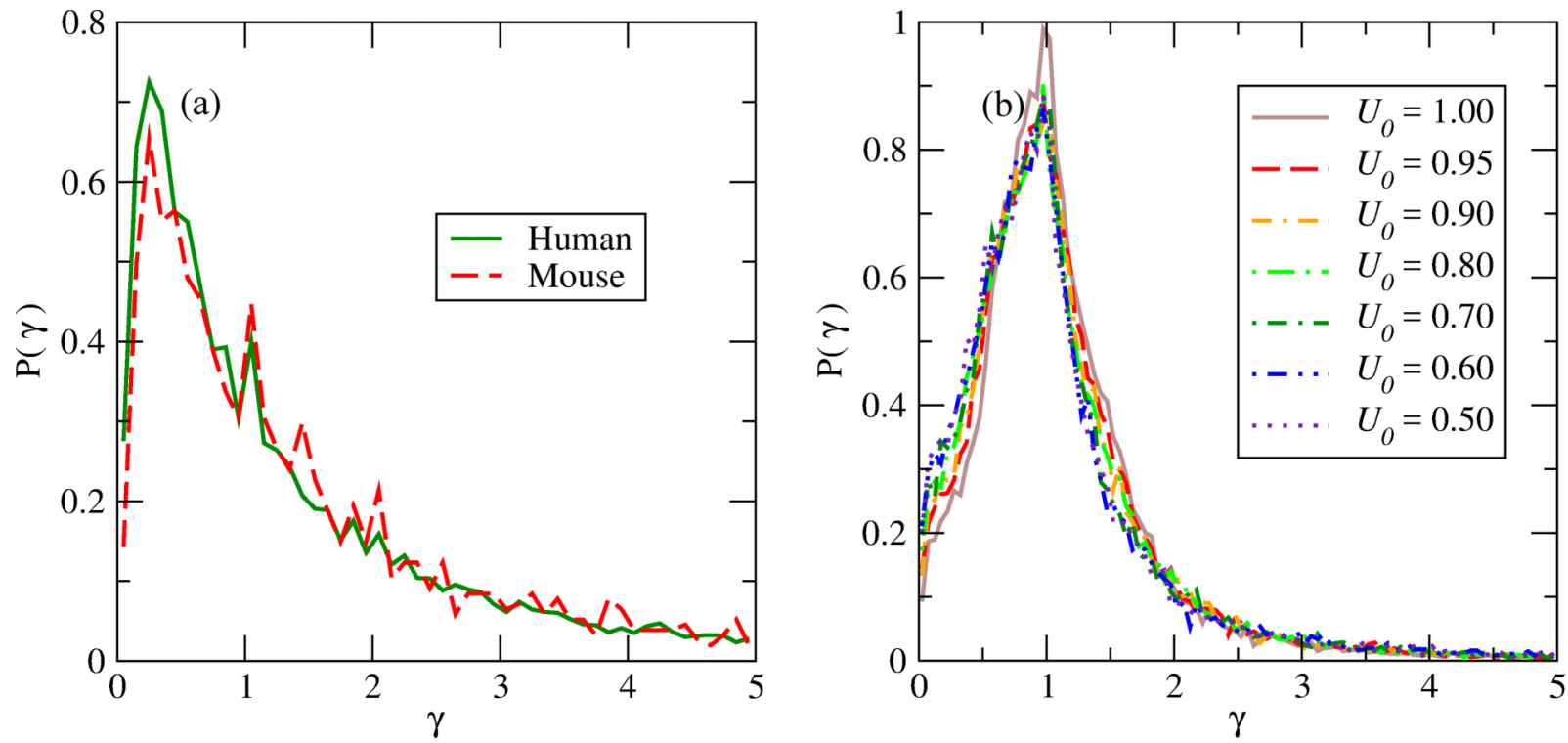

FIG. 14. Distributions for the ratio of child-to-parent length $\gamma$. (a) Distributions of $\gamma$ in mouse lung and human head and torso. (b) Distributions of $\gamma$ for several thresholds of hierarchical unbalance $U_{0}$, optimized solely for total network length $L[F(1,0 ; 1)]$.

similar to a hierarchy $\mathrm{B}$, which itself is very similar to $\mathrm{C}$. Then it is possible that $\mathrm{A}$ and $\mathrm{C}$ are less similar to each other than each is to $\mathrm{B}$, yet both are ranked higher than $\mathrm{B}$ with respect to a particular measure. This also means that optimal configurations are not always a single swap or regraft away from all near-optimal configurations, i.e., local minima are possible. The average similarity in Fig. 11(b) does not approach 1, indicating that the subtree grouping of service volumes can be very different between networks that are nearly optimal $\left(F \approx F_{\text {opt }}\right)$. Some of the stratification into distinct levels of similarity is apparent in Fig. 11(a) for the single trajectories (the "single BHC seed" and "best random seed").

\section{APPENDIX B: EFFICIENCY OF SAMPLING IN EXHAUSTIVE LANDSCAPES}

To show the proficiency of our sampling algorithm described in Sec. VB, we present results for the performance of the partial search algorithm on small networks, for which it is feasible to exhaustively search the space of configurations and specify the overall rank within the complete space. In Fig. 12(a), we show the relation directly between the ranks sampled in the partial search and the ranks in an exhaustive search over 100 realizations of tips. The exponential convergence to the optimal configuration during a partial sampling is confirmed in Fig. 12(b), which shows that the fittest configurations are sampled most heavily.

Note that in these figures the different endpoints for each curve, corresponding to different numbers of tips, are due to the different numbers of unique bifurcating hierarchies and their pairing with terminal volumes. For example, the best normalized rank for $N_{\text {tips }}=4$ is $1 /(2 \cdot 4-3) ! !=1 / 15$, while the best normalized rank for $N_{\text {tips }}=7$ is $1 / 10,395$. To emphasize this increase in possible configurations that is a result of the inclusion of additional tips, we show the distributions for the number of unique configurations after consolidation in Fig. 13(a). The variance in the number of unique configurations increases with network size, but this variance is primarily due to the addition of service volumes. In other words, degenerate branch points that lead to the same tree configuration do indeed reduce the number of unique configurations, but not by a significant fraction when compared to the additional configurations that are introduced because of an extra tip. The remaining difference is due to the arbitrary ranking of degenerate configurations, which makes a significant contribution for a small number of tips but decreases in severity as more tips (and more levels between the heart and tips) are added.

Ultimately, the reliability of the algorithm rests on the ability to determine very near-optimal configurations. In Fig. 13(b), we show the probability that the partial search finds a configuration as least as fit as a certain exhaustive ranking. Although there is no guarantee to find the fittest configuration in all cases, we do find the best configuration during the majority of searches. Furthermore, even the worst-performing partial searches appear in a narrowing margin as more tips are considered. Due to these trends that show improved sampling with more tips, we conclude that the partial search algorithm adequately determines optimal or very-near optimal configurations for our analysis.

\section{APPENDIX C: PARENT-CHILD LENGTH RATIO $\gamma$}

In Fig. 14(a) we show the network-wide distribution for the ratio of child-to-parent lengths $\gamma=\ell_{c} / \ell_{p}$ for child $c$ with parent $p$. Although there is the tendency that $\gamma<1$, some child segments have a relatively shorter parent. Although slight, an increased threshold for $U_{0}$ shifts more child segments to be shorter than their associated parent [see Fig. 14(b)]. Independent of the threshold, the nonzero variance of this distribution shows that $\gamma$ is not constant throughout the network. 
[1] A. Krogh, The supply of oxygen to the tissues and the regulation of the capillary circulation, J. Physiol. 52, 457 (1919).

[2] G. B. West, J. H. Brown, and B. J. Enquist, A general model for the origin of allometric scaling laws in biology, Science 276, 122 (1997).

[3] V. M. Savage, E. J. Deeds, and W. Fontana, Sizing up allometric scaling theory, PLoS Comput. Biol. 4, e1000171 (2008).

[4] T. Puu, On the genesis of hexagonal shapes, Networks Spatial Econ. 5, 5 (2005).

[5] G. B. West, B. Enquist, and J. Brown, A general quantitative theory of forest structure and dynamics, Proc. Natl. Acad. Sci. USA 106, 7040 (2009).

[6] V. M. Savage, L. P. Bentley, B. J. Enquist, J. S. Sperry, D. D. Smith, P. B. Reich, and E. I. von Allmen, Hydraulic trade-offs and space filling enable better predictions of vascular structure and function in plants, Proc. Natl. Acad. Sci. USA 107, 22722 (2010).

[7] J. S. Sperry, D. D. Smith, V. M. Savage, B. J. Enquist, K. A. McCulloh, P. B. Reich, L. P. Bentley, and E. I. von Allmen, A species-level model for metabolic scaling in trees i. exploring boundaries to scaling space within and across species, Funct. Ecol. 26, 1054 (2012).

[8] J. S. Andrade, Jr., H. J. Herrmann, R. F. S. Andrade, and L. R. da Silva, Apollonian Networks: Simultaneously Scale-Free, Small World, Euclidean, Space Filling, and with Matching Graphs, Phys. Rev. Lett. 94, 018702 (2005).

[9] J. Stasko, R. Catrambone, M. Guzdial, and K. McDonald, An evaluation of space-filling information visualizations for depicting hierarchical structures, Int. J. Human-Computer Stud. 53, 663 (2000).

[10] J. Kuffner and S. LaValle, Space-filling trees: A new perspective on incremental search for motion planning, in IEEE/RSJ International Conference on Intelligent Robots and Systems (IROS), 2011 (IEEE, Piscataway, NJ, 2011), pp. 2199-2206.

[11] J. Banavar, A. Maritan, and A. Rinaldo, Size and form in efficient transportation networks, Nature 399, 130 (1999).

[12] J. Banavar, J. Damuth, A. Maritan, and A. Rinaldo, Supplydemand balance and metabolic scaling, Proc. Natl. Acad. Sci. USA 99, 10506 (2002).

[13] P. Dodds, Optimal form of Branching Supply and Collection Networks, Phys. Rev. Lett. 104, 048702 (2010).

[14] Y. Huo and G. Kassab, Intraspecific scaling laws of vascular trees, J. R. Soc. Interface 9, 190 (2012).

[15] M. Kleiber, Body size and metabolism, Hilgardia 6, 315 (1932).

[16] F. Corson, Fluctuations and Redundancy in Optimal Transport Networks, Phys. Rev. Lett. 104, 048703 (2010).

[17] E. Katifori, G. J. Szöllősi, and M. O. Magnasco, Damage and Fluctuations Induce Loops in Optimal Transport Networks, Phys. Rev. Lett. 104, 048704 (2010).

[18] T. Ferrara, N.and Davis-Smyth, The biology of vascular endothelial growth factor, Endocrine reviews 18, 4 (1997).

[19] Y. Yao, S. Nowak, A. Yochelis, A. Garfinkel, and K. I. Bostöm, Matrix GLA protein, an inhibitory morphogen in pulmonary vascular development, J. Biol. Chem. 282, 30131 (2007).

[20] M. Newberry, D. Ennis, and V. M. Savage, Testing foundations of biological scaling theory using automated measurements of vascular networks, PLoS Comput. Biol. 11, e1004455 (2015).
[21] Y. Shen and J. Tolosa, The weighted fermat triangle problem, Int. J. Math. Math. Sci. 2008, 1 (2008).

[22] Peter I. Lelkes, in Mechanical Forces and the Endothelium, part of the Endothelial cell research series, edited by Gabor M. Rubanyi (Harwood Academic Publisher, Amsterdam, The Netherlands, 1999), Vol. 6, p. 291.

[23] G. Torres, A weiszfeld-like algorithm for a weber location problem constrained to a closed and convex set, arXiv:1204.1087.

[24] M. Zamir, The Physics of Coronary Blood Flow (Springer Science+Business Media, Inc., New York and Philadelphia, 2005).

[25] D. Sankoff and P. Rousseau, Locating the vertices of a steiner tree in an arbitrary metric space, Math. Program. 9, 240 (1975).

[26] F. Ronquist, M. Teslenko, P. van der Mark, D. Ayres, A. Darling, S. Höhna, B. Larget, L. Liu, M. Suchard, and J. Huelsenbeck, Mrbayes 3.2: Efficient bayesian phylogenetic inference and model choice across a large model space, Systematic Biol. 61, 539 (2012).

[27] S. Guindon, J. Dufayard, V. Lefort, M. Anisimova, W. Hordijk, and O. Gascuel, New algorithms and methods to estimate maximum-likelihood phylogenies: Assessing the performance of phyml 3.0, Systematic Biol. 59, 307 (2010).

[28] G. Weber, L. Ohno-Machado, and S. Shieber, Representation in stochastic search for phylogenetic tree reconstruction, J. Biomed. Info. 39, 43 (2006).

[29] A. Dress and M. Krüger, Parsimonious phylogenetic trees in metric spaces and simulated annealing, Adv. Appl. Math. 8, 8 (1987).

[30] L. A. Salter and D. K. Pearl, Stochastic search strategy for estimation of maximum likelihood phylogenetic trees, Systematic Biol. 50, 7 (2001).

[31] L. Gosselin and A. Bejan, Emergence of asymmetry in constructal tree flow networks, J. Appl. Phys. 98, 104903 (2005).

[32] D. Hu and D. Cai, Adaptation and Optimization of Biological Transport Networks, Phys. Rev. Lett. 111, 138701 (2013).

[33] J. Banavar, M. Moses, J. Brown, J. Damuth, A. Rinaldo, R. Sibly, and A. Maritan, A general basis for quarter-power scaling in animals, Proc. Natl. Acad. Sci. USA 107, 15816 (2010).

[34] H. Meinhardt, Morphogenesis of lines and nets, Differentiation 6, 117 (1976)

[35] G. Serini, D. Ambrosi, E. Giraudo, A. Gamba, L. Preziosi, and F. Bussolino, Modeling the early stages of vascular network assembly, EMBO J. 22, 1771 (2003).

[36] A. Gamba, D. Ambrosi, A. Coniglio, A. de Candia, S. Di Talia, E. Giraudo, G. Serini, L. Preziosi, and F. Bussolino, Percolation, Morphogenesis, and Burgers Dynamics in Blood Vessels Formation, Phys. Rev. Lett. 90, 118101 (2003).

[37] D. Manoussaki, S. R. Lubkin, R. B. Vemon, and J. D. Murray, A mechanical model for the formation of vascular networks in vitro, Acta Biotheoretica 44, 271 (1996).

[38] R. M. H. Merks and J. A. Glazier, Dynamic mechanisms of blood vessel growth, Nonlinearity 19, C1 (2006).

[39] R. M. H. Merks and P. Koolwijk, Modeling morphogenesis in silico and in vitro: Towards quantitative, predictive, cell-based modeling, Math. Model. Nat. Phenom. 4, 149 (2009).

[40] J. Keelan, E. Chung, and J. Hague, Simulated annealing approach to vascular structure with application to the coronary arteries, Roy. Soc. Open Sci. 3, 150431 (2016). 
[41] M. Zamir, Nonsymmetrical bifurcations in arterial branching, J. Gen. Physiol. 72, 837 (1978).

[42] M. Zamir, The Physics of Pulsatile Flow (Springer, New York, 2005).

[43] T. Wischgoll, J. S. Choy, and G. S. Kassab, Extraction of morphometry and branching angles of porcine coronary arterial tree from ct images, Am. J. Physiol. Heart Circ. Physiol. 297, H1949 (2009).

[44] A. N. Zachos, An analytical solution of the weighted fermattorricelli problem on the unit sphere, arXiv:1408.6495.

[45] A. N. Zachos, Exact location of the weighted fermat-torricelli point on flat surfaces of revolution, Results Math. 65, 167 (2014).
[46] Y. Mileyko, H. Edelsbrunner, C. A. Price, and J. S. Weitz, Hierarchical ordering of reticular networks, PLoS ONE 7, e36715 (2012).

[47] A. B. Herman, V. M. Savage, and G. B. West, A quantitative theory of solid tumor growth, metabolic rate and vascularization, PLoS One 6, e22973 (2011).

[48] V. M. Savage, A. B. Herman, G. B. West, and K. Leu, Using fractal geometry and universal growth curves as diagnostics for comparing tumor vasculature and metabolic rate with healthy tissue and for predicting responses to drug therapies, Discrete Contin. Dyn. Syst. B 18, 1077 (2013). 